\title{
Implementation of the FilmArray ME panel in laboratory routine using a simple sample selection strategy for diagnosis of meningitis and encephalitis
}

Susanne Pfefferle ${ }^{*}$ (D), Martin Christner, Martin Aepfelbacher, Marc Lütgehetmann and Holger Rohde

\begin{abstract}
Background: Infectious meningitis is a serious disease and patient outcome relies on fast and reliable diagnostics. A syndromic panel testing approach like the FilmArray ME can accelerate diagnosis and therefore decrease the time to pathogen specific therapy. Yet, its clinical utility is controversial, mainly because of a remaining uncertainty in correct interpretation of results, limited data on its performance on clinical specimens and its relatively high costs. The aim of this study was to analyze clinical performance of the assay in a real life setting at a tertiary university hospital using a pragmatic and simple sample selection strategy to reduce the overall cost burden.

Methods: Over a period of 18 months we received 4623 CSF samples (2338 hospitalizations, 1601 individuals). FilmArray ME analysis was restricted to CSF-samples with a high pretest probability of infectious meningitis, e.g. positive Gram-stain, samples in which leukocytes and/or bacteria were evident or urgent suspicion of infection was communicated by clinicians. $N=171$ samples matched to our risk criteria and were subjected to FilmArray ME analysis. Those samples were also analyzed by reference methods: culture only $(n=45)$, PCR only $(n=20)$ or both methods $(n=106)$.

Results: 56/171 (32.75\%) were FilmArray ME positive. Bacterial pathogens were detected in 30/56 (53.57\%), viral pathogens were detected in 27/56 (48.21\%) and yeast DNA was detected in 1/56 (1.79\%) of positive samples. Double detection occurred in 2/56 samples. In 52/56 (92.86\%) FilmArray ME positive samples, results could be confirmed by the reference assays (sensitivity $=96.30 \%$, specificity $=96.58 \%$ ).

Conclusion: The FilmArray ME assay is a fast and reliable diagnostic tool for the management of infectious meningitis and can easily be implemented in routine diagnostic workflows. However, correlation of test results and underlying clinical symptoms requires experienced users and the awareness of potentially false negative or false positive results. Moreover, considering the need for antimicrobial susceptibility testing, the use of molecular tests as a stand-alone diagnostic cannot be recommended.
\end{abstract}

Keywords: Film Array ME, Rapid diagnostic, Syndromic panel testing, Infectious meningitis

\footnotetext{
* Correspondence: s.pfefferle@uke.de

Institut für Medizinische Mikrobiologie, Virologie und Hygiene,

Universitätsklinikum Hamburg-Eppendorf, Martinistraße 52, 20246 Hamburg,

Germany
}

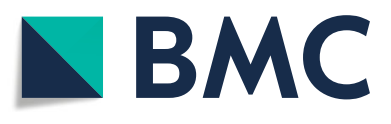

(c) The Author(s). 2020 Open Access This article is distributed under the terms of the Creative Commons Attribution 4.0 International License (http://creativecommons.org/licenses/by/4.0/), which permits unrestricted use, distribution, and reproduction in any medium, provided you give appropriate credit to the original author(s) and the source, provide a link to the Creative Commons license, and indicate if changes were made. The Creative Commons Public Domain Dedication waiver (http://creativecommons.org/publicdomain/zero/1.0/) applies to the data made available in this article, unless otherwise stated. 


\section{Background}

Infectious meningitis is a serious threat with high morbidity and mortality rates [1-4]. In bacterial meningitis, patient outcome can be optimized through immediate antibiotic treatment, thus fast and reliable diagnostic can be crucial $[2,5]$. Direct identification of viral, bacterial and fungal pathogens in cerebrospinal fluid (CSF) by molecular testing methods may substantially decrease the time to pathogen specific therapy. However, CSF is a delicate clinical sample, limiting the diagnostic possibilities by its low quantity.

The Biofire FilmArray ME Panel provides for a simultaneous multiplex testing of a comprehensive panel of 14 pathogens using a minimal amount of CSF (e.g. $200 \mu \mathrm{l})$. This sample-to-answer system was FDA-cleared in 2015. However, in the multicenter evaluation study, reported detection rates for bacterial pathogens typically found in infectious meningitis cases were surprisingly low. Most notably, $N$. meningitides, a worldwide leading meningitis pathogen, and L. monocytogenes detected in none of the samples [6]. Meanwhile, clinical data on the performance of the FilmArray ME was reported by various users worldwide [7-13] with diagnostic specificity and sensitivity in good concordance to the multicenter study Admittedly, both false positive and false negative detections have been reported throughout the studies, with one example of an erroneously diagnosed HSV-1 meningitis in a patient with an underlying tuberculous meningitis [14] and one study indicating a potential of missing viral infections in a pediatric cohort [10]. Aside from the inarguably higher costs of multiplex molecular detection methods compared to standard laboratory procedures like bacterial culture, the remaining uncertainty in interpretation of results is a key point in the controversial discussion concerning the clinical utility of such syndromic testing approaches in infectious meningitis/ encephalitis [15]. Nevertheless, there is agreement regarding the substantially decreased time-to-result exhibiting a potential benefit for patient outcome whenever involving an adaptation of antibiotic treatment. Yet, studies on prospective clinical specimens indicating the right patient population or determining the ideal testing approach are missing [15]. We report our real-life experience of the implementation of the FilmArray ME panel in addition to available conventional cultural and molecular diagnostics from CSF in daily laboratory routine in a university hospital setting. In order to avoid unnecessary and cost intensive rapid diagnostics, FilmArray ME analysis was restricted to CSF-samples with a high pretest probability of infectious meningitis. Risk for infection was prospectively assessed by Gram-stain, and only samples in which leukocytes and/or bacteria were evident or urgent suspicion of infection was communicated by clinicians were subsequently analyzed with the Film Array ME assay.

\section{Methods}

\section{Clinical specimens and selection strategy}

The study was conducted over a period of approximately 18 months (September 2015 through February 2017) in our laboratory. CSF samples demonstrating abnormality in Gram-stain (e.g. leucocytes and/or bacteria visible) and CSF samples of patients with urgent suspicion of infection as communicated by clinicians were selected for additional FilmArray ME analysis (see Fig. 1) Gram stain abnormalities were chosen for ensuring the most rapid decision-making. The FilmArray ME panel could not be ordered directly by clinicians, yet results were reported.

\section{Ethics}

This work was conducted in accordance with $\$ 12$ of the Hamburg hospital law ( $\$ 12$ HmbKHG).

\section{Primary bacterial culture and gram-stain}

CSF samples were centrifuged for $10 \mathrm{~min}$ at $3000 \mathrm{rpm}$ at room temperature. Supernatant was used for Bacillus subtilis inhibition-assay for preliminary assessment of presence of antibiotics [16]. With one drop of sediment, a smear was generated on glass slides. Gram-staining was performed after drying and fixation of smear. Equal amounts of sediment were plated onto blood agar plates, chocolate agar plates and Sabouraud agar plates and incubated for $48 \mathrm{~h}$ at $35-37^{\circ} \mathrm{C}$ with $5 \% \mathrm{CO}_{2}$, one drop of CSF sediment was inoculated in thioglycollate medium (thioglycollate broth) and incubated at $37^{\circ} \mathrm{C}$. All Plates and broth are inspected at least once daily for bacterial growth. Species identification relied on MALDI-TOF technique combined with classical biochemical identification methods.

\section{NAAT analysis}

Nucleic acid extraction (RNA and DNA) was performed on a QIAsymphony SP/AS instrument with the QIAsymphony DSP Virus/ Pathogen Mini kit using $200 \mu \mathrm{l}$ of native CSF sample. During extraction process, universal PCR inhibition control (DNA/RNA) was added. Elution volume was $60 \mu \mathrm{l}$. Quantitative real-time-PCR reaction was set-up using 5-10 $\mu \mathrm{l}$ of eluate, reverse-transcription, amplification and analysis were performed on a LightCycler 480 instrument. The quantitative real-time PCRs used in CSF diagnostic are laboratory developed tests $(L D T s)$, specific primers and probes are designed based on sequences and assays published elsewhere [17-31]. Table 1 gives a list of LDTs used for detection of pathogens included in the FilmArray ME with underlying references. For specific detection of human herpesvirus 1 and human herpesvirus 2, commercially available RealStar ${ }^{\circledR}$ PCR Kit (ATD) was used for verification of results. For molecular detection of BKV, JCV and EBV (human herpesvirus 4) in CSF samples, also molecular LDTs 


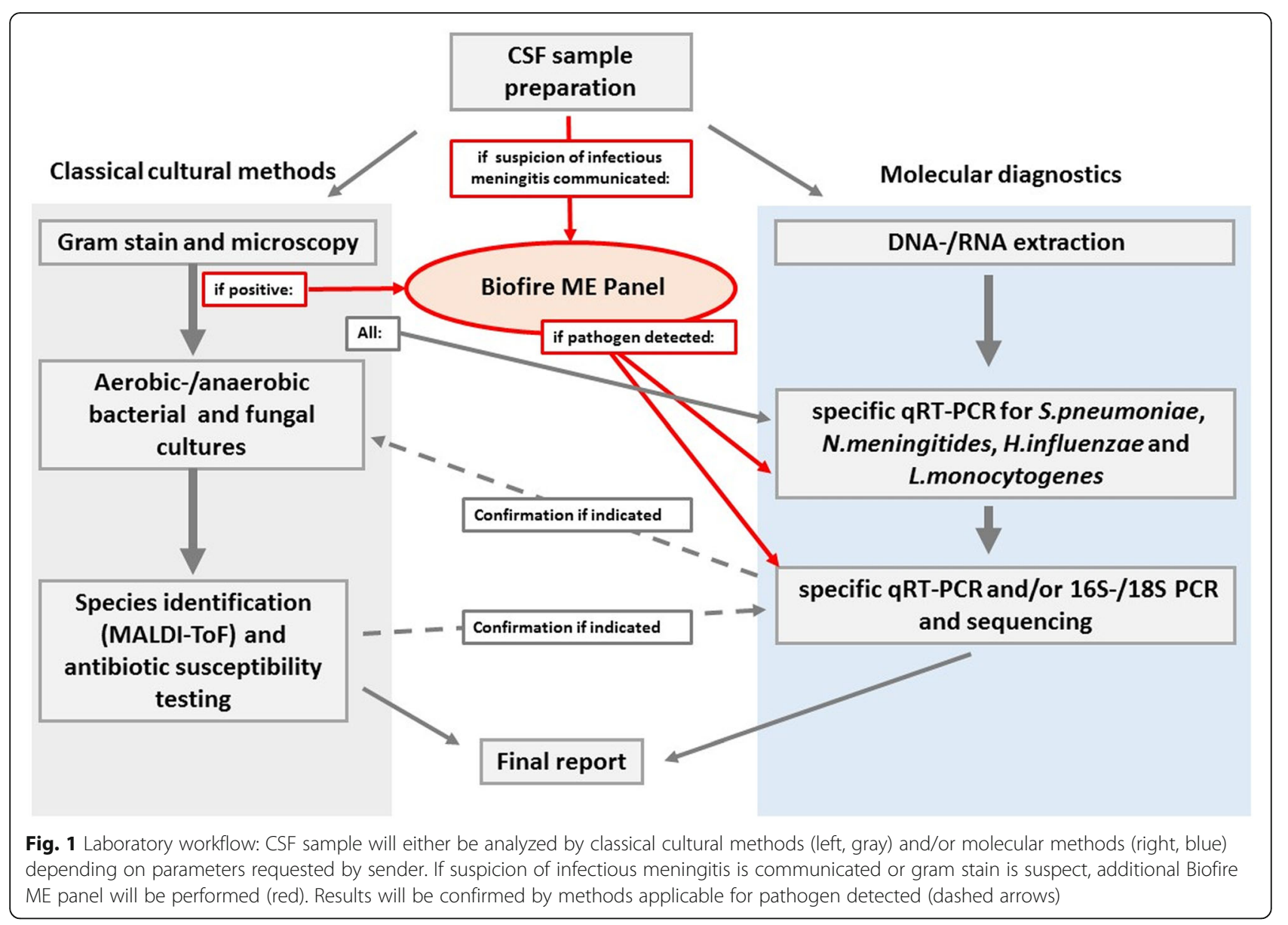

were used [23, 28, 29]. For universal molecular detection of bacterial 16S rRNA and mycotic 18S rRNA, SepsiTest $^{\text {tim }}$-UMD (Molzym) was used according to the manufacturer's instructions. Briefly, $1000 \mu \mathrm{l}$ of CSF were used for nucleic acid extraction, amplification and melting curve analysis was performed on a LightCycler 2.0 instrument.. The sequence of amplicons was determined using Sanger sequencing. Sequences were analyzed with SepsiTest BLAST (www.sepsitest-blast.de/en/index.html) and the BLAST tool of NCBI (www.ncbi.nlm.nih.gov/ blast).

\section{FilmArray ME panel}

FilmArray ME Panel testing was performed according to the manufacturer's instructions. Briefly, $200 \mu \mathrm{l}$ of CSF specimen were mixed with sample buffer by inverting. Sediment leftover or supernatant was used if samples were entirely processed for primary culture (off-label use). Hydration solution was injected into the FilmArray ME pouch to rehydrate the freeze-dried reagents, sample mixture was thereafter injected. The pouch was transferred to the instrument and run was initiated. Nucleic acid purification, reverse transcription, multiplex PCR and melting curve analysis are performed automatically, with a runtime of about $1 \mathrm{~h}$. Test reports are generated by instrument software.

\section{Results}

Over a period of 18 months we received a total of $n=$ 4623 CSF samples (2338 hospitalizations, 1601 individuals), including samples sent for both molecular diagnostic and bacterial culture as well as samples sent only for either purpose. In detail, bacterial culture was performed in $n=2682$ samples, molecular diagnostic was performed in $n=1891$ samples and $n=50$ samples were sent for serology purpose. The overall positivity rate was $5.97 \%$ with positive results in 137/2682 samples analyzed by culture $(5.11 \%)$ and $139 / 1891$ positive results in samples analyzed by molecular methods (7.35\%). More details are given in supplementary Tables 1 and 2 .

Overall, $n=171$ samples matched to our risk criteria and were subjected to FilmArray ME analysis. Of those, $n=117$ samples had abnormality in gram-stain $(n=116$ samples with leukocytes, one samples with bacteria seen without evidence for leukocytes) and $n=54$ samples were selected as suspicion of infectious meningitis was communicated by clinicians. Of note, in 24/54(44.44\%) of samples selected upon clinical request, gram stain and primary 
Table 1 Laboratory procedures for each pathogen included in the Biofire FilmArray ME panel are listed ( $X=$ performed regularly). Primary culture included cultivation of CSF samples at $35-37^{\circ} \mathrm{C}$ with $5 \% \mathrm{CO}_{2}$, all samples were plated on blood agar plates (BAB), chocolate agar (CHOC) and sabouraud agar, and were incubated in fluid thioglycollate medium (thioglycollate broth), respectively. In case of CSF-samples sent for detection of yeast and bacterial pathogens, gram stain and primary culture are performed routinely, NAT analysis is performed if mandatory (e.g. verification of bacteria seen in gram stain). Additionally, bacterial 165 rRNA genes and mycotic 18S rRNA sequences are detected via commercially available SepsiTest ${ }^{\mathrm{TM}}$-UMD (Molzym) upon request. For all viral pathogens, primarily quantitative real-time PCR was performed. In these samples, gram stain was only performed upon request $\left({ }^{* 1}\right)$. For Human herpesvirus 1 and human herpesvirus 2, commercially available RealStar ${ }^{\circledR}$-PCRs (Altona Diagnostics) were performed for confirmation if indicated. Diagnostic PCRs for CSF diagnostic in our laboratory are laboratory developed tests ("LDTs"), all LDTs are quantitative real-time PCRs using specific primers and probes. Design of LDTs is based on references given into parentheses

\begin{tabular}{|c|c|c|c|c|}
\hline & Pathogen & Gram stain & Primary culture & NAT \\
\hline \multirow[t]{6}{*}{ Bacteria } & Escherichia coli K1 & $x$ & $x$ & LDT (Diaz, Waller [21]/ SepsiTest ${ }^{\text {TM_UMD }}$ \\
\hline & Haemophilus influenzae & $x$ & $x$ & LDT (Abdeldaim, Stralin [18])/SepsiTest ${ }^{\mathrm{TM}}{ }^{-U M D}$ \\
\hline & Listeria monocytogenes & $x$ & $x$ & LDT (Le Monnier, Abachin [25])/SepsiTest ${ }^{\text {TM}}$-UMD \\
\hline & Neisseria meningitidis & $x$ & $x$ & LDT (Abdeldaim, Stralin [18])/SepsiTest ${ }^{\text {TM}}{ }^{-U M D}$ \\
\hline & Streptococcus agalactiae & $x$ & $x$ & LDT (Diaz, Waller [21])/SepsiTest ${ }^{\mathrm{TM}}-$ UMD \\
\hline & Streptococcus pneumoniae & $x$ & $x$ & LDT (Stralin, Herrmann [30])/SepsiTest ${ }^{\mathrm{TM}}$-UMD \\
\hline \multirow[t]{7}{*}{ Viruses } & Cytomegalovirus & $x^{* 1}$ & & LDT (Khansarinejad, Soleimanjahi [24]) \\
\hline & Enterovirus & $x^{* 1}$ & & LDT (Dierssen, Rehren [22]) \\
\hline & Herpes simplex virus 1 & $X^{* 1}$ & & LDT (Meylan, Robert [26])/RealStar ${ }^{\circledast}$ HSV \\
\hline & Herpes simplex virus 2 & $X^{* 1}$ & & LDT (Meylan, Robert [26])/RealStar ${ }^{\oplus}$ HSV \\
\hline & Human herpesvirus 6 & $x^{* 1}$ & & LDT (Cassina, Russo [20]) \\
\hline & Human parechovirus & $X^{* 1}$ & & LDT (Benschop, Molenkamp [19]) \\
\hline & Varizella zoster virus & $X^{* 1}$ & & LDT (Pollak, Dovrat [27]) \\
\hline Yeast & Cryptococus neoformans/gattii & $x$ & $x$ & LDT (Veron, Simon [31])/SepsiTest ${ }^{\text {TMM-UMD }}$ \\
\hline
\end{tabular}

bacterial culture was not performed as suspicion for viral infection was communicated. 6/30 samples of that subset had positive gram stain (20.00\%), whereas $25 / 30$ samples had negative gram stain (83.33\%). Overall, gram stain was performed in $147 / 171$ samples. Leucocytes were seen in $122 / 147$ (82.99\%) samples, bacteria in 15/147 (10.20\%) samples and yeast in $1 / 147(0.68 \%)$ of those samples.

Samples analyzed by FilmArray ME were also tested by reference methods: culture only $(n=45)$, molecular analysis only $(n=20)$ or both methods $(n=106) .56 / 171$ $(32.75 \%)$ were FilmArray ME positive. Bacterial pathogens were detected in 30/56 (53.57\%) and viral pathogens were detected in 27/56 (48.21\%) of positive samples. Additionally, Cryptococcus neoformans was detected in 1/56 samples (1.79\%). Double detection occurred in two samples. An overview of pathogens detected is given in Fig. 2.

Within the subset of samples selected for leucocytes seen in gram stain $(n=116)$, positive Film Array ME results were obtained in 44/116 samples (positivity rate $37.93 \%$, sensitivity $=99.45 \%$, specificity $=10.96 \%$ ). Organisms have been seen in 16/117 samples selected for positive gram stain (bacteria in 15/117 samples (12.82\%), yeast in $1 / 117$ samples $(0.85 \%$, positivity rate $=13.63 \%$, sensitivity $=22.73 \%$, specificity $=91.78 \%$ ). Within the subset of samples selected upon request by clinicians, $(n=54)$, positive Film Array ME results were obtained in
44/54 samples (positivity rate $=22.22 \%$, sensitivity $=$ $91.67 \%$, specificity $=97.62 \%)$.

\section{Clinical performance}

52/56 positive FilmArray ME results could be confirmed by the reference assays (sensitivity $=96.30 \%$, specificity $=$ $96.58 \%$, PPV $=92.86 \%$ ). In four samples that were positive for S. pneumoniae, E. coli, S. agalactiae or HSV-1 in FilmArray ME panel, the result could not be confirmed by a reference method (Fig. 2, light grey bars). For the three samples positive for bacterial pathogens E.coli, S. pneumonia and S. agalactiae, primary culture showed no bacterial growth after incubation period. Specific real-time PCR was performed in the two samples positive for S. pneumoniae and $S$. agalactiae, but no signal was obtained. Also, no bacterial 16S-rRNA was detected in the sample positive for S. pneumoniae in SepsiTest ${ }^{\oplus}$-UMD (Molzym). Residual material of the two samples positive for E. coli $K 1$ and $S$. agalactiae was not sufficient for additional 16S-rRNA amplification, as proper DNA extraction for this method requires a high input volume (e.g. $1000 \mu \mathrm{l}$ of CNS liquid). No specific real-time PCR assay for detection of E.coli K1 was applied. One sample that was FilmArray positive for HSV-1 could not be confirmed by two independent molecular detection methods. Neither our laboratory developed real-time PCR assay, nor a commercially available 


\section{Cryptococcus neoformans}

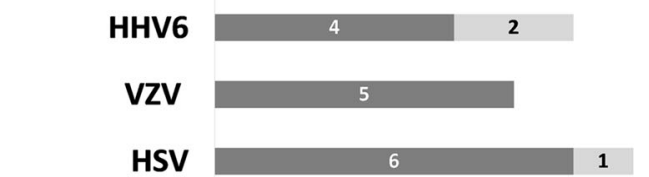

Parechovirus

Enterovirus

E.coli

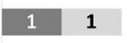

Listeria monocytogenes

Streptococcus agalactiae

Haemophilus influenzae

Neisseria meningitidis

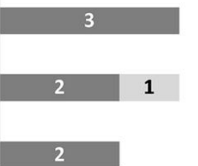

Streptococcus pneumoniae

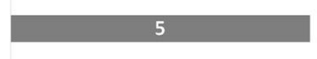

\section{n= Filmarray ME Panel positive \\ 6 \\ $8 \quad 10$ \\ 12 \\ 14 \\ 16 \\ 18 \\ 20}

Fig. 2 Summary of FilmArray ME positive result (dark gray). Light gray bars indicate disconcordant results, e.g. positive FilmArray ME results that could neither be confirmed by standard cultural methods nor by molecular testing. For $S$. pneumoniae, one sample could not be confirmed by specific PCR nor by cultural methods, whereas $n=16$ samples were confirmed by specific PCR and $n=12$ of FilmArray ME positive samples were confirmed by both methods. For S. agalactiae, one of the FilmArray ME positive samples could neither be confirmed by cultural methods nor by specific PCR. For E.coli K1 detected in FilmArray ME also no confirmation was obtained by any method. One sample was designated HSV-1 positive by FilmArray ME, but no such pathogen could be detected by two different specific quantitative real-time PCRs. HHV- 6 was detected in four samples by Film Array ME, two of those yielded a double hit (positive for HHV-6 and VZV or H. influenzae and HHV-6, respectively).

Conventional assays confirmed presence of VZV and H. influenzae, whereas HHV-6 was not found by specific PCR

real-time PCR assay yielded a positive result (see Table 1 for assay details).

Two samples (1\%) revealed negative FilmArray results, but were positive for $S$. pneumoniae and Parechovirus by specific in-house PCRs, respectively (NPV =98.26\%). In two samples, FilmArray analysis detected two pathogens ( $V Z V$ and $H H V-6$, and $H$. influenzae and $H H V-6$, respectively). Presence of $V Z V\left(1.4 \times 10^{4}\right.$ copies $\left./ \mathrm{ml}\right)$ and $H$. influenzae was confirmed by specific real-time PCRs, but conventional assays were unable to detect $H H V-6$, and these results were thus regarded as false-positive. In 10 samples, pathogens not found by the FilmArray ME panel were detected by culture (Bacillus sp., CoNS, S. aureus, $K$. pneumoniae) or PCR (BK virus, Streptococcus spp.).

\section{Estimation of the impact of our risk-assessment driven} sample selection strategy

Results were compared to the multicenter evaluation study [6] and three other studies [7, 32, 33], that reported Film Array performance data on a high quantity of CSF samples tested $(n=1560, n=969, n=705$ and $n=253$ CSF samples analyzed by Film Array ME, respectively). Figure 3 illustrates positivity rates and pathogen composition in CSF samples analyzed by different studies.

\section{Discussion}

We here report on our real-life experience of the implementation of the FilmArray ME panel into a diagnostic laboratory in a German university hospital setting. We implemented a simple sample selection strategy in our workflow: FilmArray ME analysis was restricted to CSFsamples with a high pretest probability of infectious (bacterial) meningitis. Decision was made in our laboratory based on gram stain and/or urgent suspicion of infectious meningitis communicated by clinicians, e.g. neither the clinical decision for lumbar puncture was affected, nor was explicit ordering of the assay permissible. 


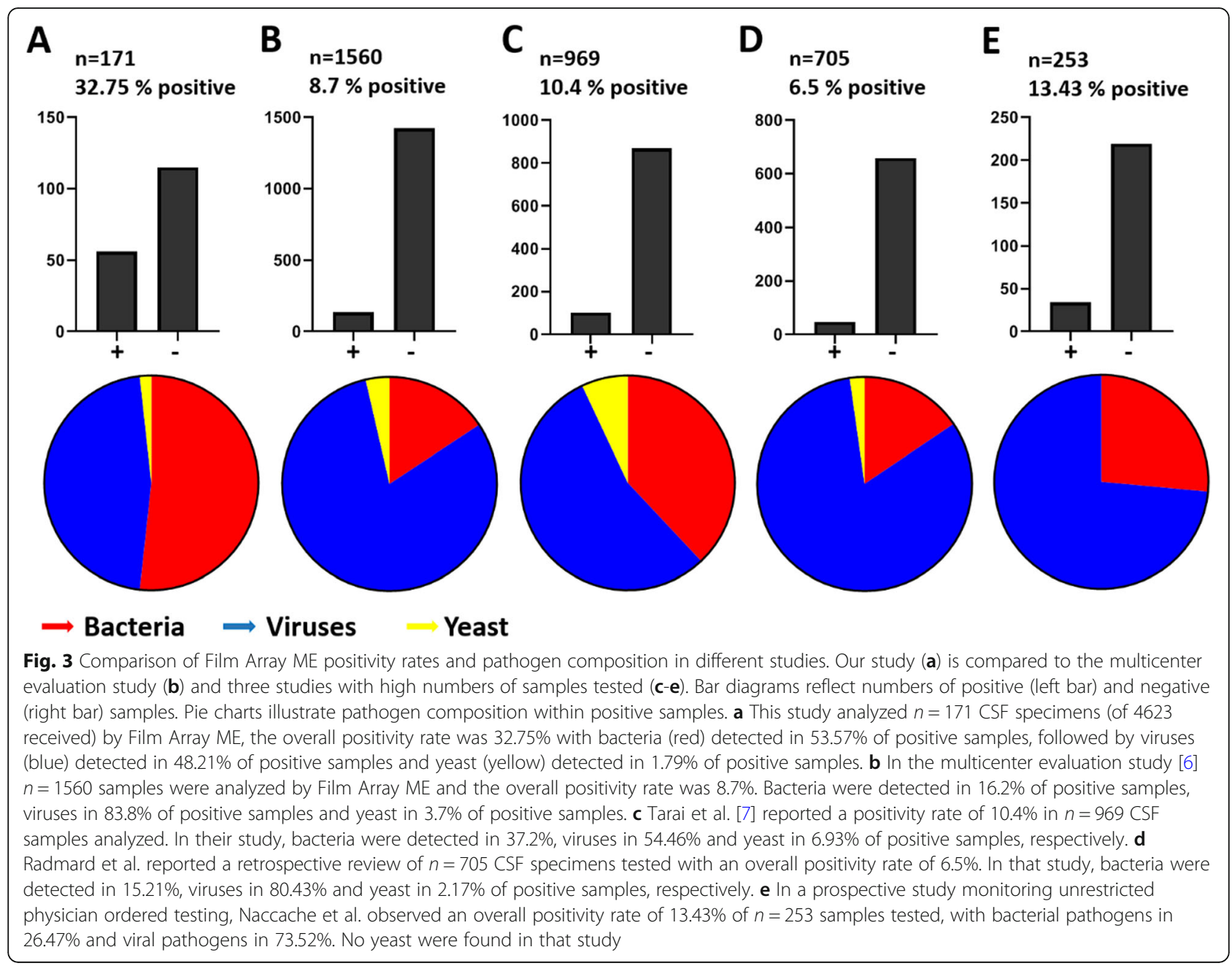

Using this selection criteria we selected 171/4623 CSF specimens for additional syndromic molecular testing. Overall we observed positive results in 56/171 (32\%), which is higher than in other published studies $[6,7,9-$ $12,34-38]$ and substantially higher compared to the multicenter evaluation study [6] and three other studies $[7,32,33]$, that reported positivity rates of under $15 \%$ $(p<0.0001)$. In addition, we could increase the amount of bacterial pathogens to $53 \%$ of the specimens, again this is more than in other published studies that observed positive ranges for bacterial pathogens between 15 and $37 \%$ [6, 7, 32, 33]. Indeed, bacteria were the most abundant pathogens detected in our study $(53.57 \%$ of positive samples), followed by viruses detected in $48.21 \%$ of positive samples. In contrast, in the comparator studies viruses were detected most frequently (83.8 -54.46\% of positive samples) followed by bacteria (37.62 - 15.21\% of positive samples, $p<0.0001$ ).

In concordance with other studies [6, 7, 9-12, 34-38], S. pneumoniae $(17 / 30)$ was the most frequent bacterial pathogen detected by the FilmArray ME assay.
Additionally, in 10/30 specimens rapid detection of $N$. meningitides $(n=5), H$. influenzae $(n=2)$ or $L$. monocytogenes $(n=3)$ was important for optimal clinical management including rapid implementation of post exposure treatments in contact persons (antibiotic prophylaxis and/or vaccination).

Analytically we observed good concordance (52/56 positive results could be confirmed) between the FilmArray ME panel results and our comparator assays. Only in two of the samples the FilmArray ME panel yielded a discordant negative result compared to the local reference methods. Namely, in each of one sample S. pneumoniae and Parechovirus were detected by specific in-house PCRs. Although it might be crucial to detect a bacterial or viral meningitis in the individual case, our data confirm the high negative predictive value of the multiplex PCR assay that has been emphasized by others [33].

In four samples, potentially false positive results of FilmArray ME panel occurred, including three samples tested positive for bacterial pathogens by multiplex PCR 
that remained culture negative. Among the reasons for failure of primary culture is antibiotic administration prior to lumbar puncture [39]. In the three disconcordant samples, bacterial growth inhibitors (such as antibiotics) were found only in the two samples positive for $E$. coli and S. pneumoniae. One sample positive for S. agalactiae in FilmArray E panel showed no inhibition of growth in B. subtilis inhibition assay. Notably, culture negative yet PCR positive infectious meningitis cases caused by $S$. agalactiae have been described previously, underlining the importance of molecular diagnostic for optimization of patient management [40-42]. Otherwise, it cannot be excluded that in our sample, FilmArray ME detection of S.agalactiae was indeed false positive and the communication of the test result might have led to a dispensable antibiotic administration.

In our study, one sample remained disconcordant for $H S V$-1. Beside the probable useless administration of Acyclovir following a false positive $H S V-1$ result, one should also consider the questionable relevance of Herpesviridae detection in CSF in general. As these viruses feature life-long latency after primary infection, subclinical reactivation with replication can be seen in conjunction with different underlying clinical conditions $[43,44]$. Thus, in a worst case scenario, Herpesviridae detection in multiplex PCR testing might lead to delayed diagnosis of the actual underlying disease, as described by Gomez et al. for a patient suffering from tuberculous meningitis [14].

The herpesvirus $H H V-6$ is included in the FilmArray ME panel. This virus exhibits not only latency and also has the potential of chromosomal integration. Therefore, careful interpretation of $H H V-6$ detection in FilmArray ME panel is mandatory. The clinical diagnosis should generally not be made by molecular detection of HHV-6 in CSF alone $[45,46]$. As this study focused on the implementation of the assay into the laboratory workflow, no clinical data was analyzed. The relevance of $H H V-6$ detection in our study remains unclear in its consequences for patient management and outcome.

An obvious limitation of any syndromic molecular panel testing approach is the limited number of pathogens included into the panel. Even though the FilmArray ME panel includes a broad range of pathogens, overreliance on negative results might be crucial especially in geographical regions with unusual etiologic agents of meningitis this might yield to ineffective testing [7]. In our study, we detected pathogens that are not included in the panel by comparator assays in 10 samples: Bacillus sp., CoNS, S. aureus, and K. pneumoniae grew on culture media, whereas BK virus and Streptococcus spp. were detected by PCR. Albeit the questionable clinical relevance of Bacillus sp., Streptococcus spp. and CoNS in a CSF sample, detection of $S$. aureus, K. pneumoniae and BK-Virus may account for serious infections. This underlines, that reasonable and effective CNS diagnostic should combine classical cultural and molecular methods, rather than focus on one test system alone.

It has been proposed recently that implementation of the FilmArray ME Panel in routine diagnostic may help for cost saving in direct antimicrobial utilization and might decrease diagnostic costs even with uncontrolled routine availability of the assay $[47,48]$.

However, both Naccache et al. and Radmard et al. reported massive overutilization of the test, when no eligibility criteria was implemented [32,33]. In the latter study, more than one third of samples analyzed were taken from patients without suspicion for infectious meningitis/encephalitis. The authors advice against potential overreliance of test results and suggest restriction strategies within the scope of diagnostic stewardship programs [49]. Corroborate findings were reported by Tan et al., who describe a potential overutilization of the assay in children, but yet point out the benefits of early diagnosis of a viral etiology, especially in terms of antibiotic usage [50].

Eichinger et al. reported their findings of implementation of the FilmArray ME panel as a POCT in children with suspected meningitis in a children hospital [51]. They highlight that though the availability of rapid diagnostic tests enhances administration of specific treatment and thus reduces inadequate usage of antibiotics, a structured approach in clinical implementation of the assay is needed. Taken together, there is agreement that restriction of the assay might be helpful for enhancing its clinical utility. In a point-counterpoint discussion [15] the pros and cons of syndromic testing approaches in CSF specimens in general have been summed up. Despite their contradictory positions on the use of the FilmArray ME panel in diagnostic laboratory routine, the authors agree on the fact that it ensures reduced turn-around time of molecular results and therefore might be beneficial for patient outcome. Yet, the appropriate patient population for testing still has to be identified and more data on the performance of the assay on clinical specimens are needed to evaluate the ideal approach for testing.

Our implementation approach is independent from approval by infectious disease specialists and it does not involve any ordering modifications. The latter opportunities have been proposed for diagnostic stewardship programs [49], however, implementation of such criteria might slow-down the whole workflow. Since one major benefit of molecular multiplex testing is the fast generation of results with high negative predictive values [33], any retardation might also be discussed controversially. Therefore we believe that our restriction approach can be an easy and effective alternative. 


\section{Limitations of the study}

We report our real-life experience of the implementation of the FilmArray ME panel into our laboratory workflow, no sample randomization was done. Clinical specimens were tested prospectively, yet our selection strategy might exhibit a selection bias. Notably, sample selection strategy was mainly based on gram-stain abnormalities ensuring on-spot decision making, but not representing the ideal method for leucocyte detection. Furthermore, we did not assess all samples by all comparator assays, in particular for samples that were not selected for FilmArray ME analysis, diagnostic was performed according to orders and no additional assays were performed. Thus, we cannot rule out, that pathogens have been overlooked in some samples. Moreover, no clinical data was analyzed, hence effects on patient outcome or antibiotic utilization in our study remain unclear. Nevertheless we believe that our data gain new insights in how syndromic panel testing may be implemented into laboratory routine and therefore may help to identify the ideal approach to ensure its clinical utility.

\section{Conclusion}

The FilmArray ME is a useful tool for fast and reliable diagnostic of infectious meningitis in a real life diagnostic setting and can be easily implemented in routine diagnostic workflows. Beyond that, our strategy of riskassessment driven selection of samples might help to avoid unnecessary testing if bacterial pathogens are suspected. However, correlation of test results and underlying clinical symptoms requires experienced users and the awareness of potentially false negative or false positive results. Moreover, considering the need for antimicrobial susceptibility testing, the use of molecular tests as stand-alone diagnostic cannot be recommended.

\section{Supplementary information}

Supplementary information accompanies this paper at https://doi.org/10. 1186/s12879-020-4904-4

Additional file 1: Table S1. Overview of analytical methods performed and detection rates in $n=4623$ CSF samples received.

Additional file 2: Table S2. Overview of pathogens detected by different methods. Numbers of pathogens detected by routine diagnostic procedures, numbers detected by Film Array ME Panel as well as confirmatory results are given.

\section{Abbreviations}

BAB: Blood-agar plates; BKV: BK-Polyomavirus; CHOC: Chocolate-agar plates; CSF: Cerebrospinal fluid; HSV: Human herpesvirus; JCV: JC-Polyomavirus; PCR: Polymerase chain reaction

\section{Authors' contributions}

Conceived and designed the study: SP, ML, MC, MA, HR. Analyzed the data: SP, ML. Wrote the paper: SP. All authors have read and approved the manuscript.

\section{Funding}

Not applicable.

Availability of data and materials

Data used and/or analyzed are available from the corresponding author upon reasonable request.

\section{Ethics approval and consent to participate}

This work was conducted in accordance with $\S 12$ of the Hamburg hospital law ( $\$ 12 \mathrm{HmbKHG}$ ), e.g. no ethic approval is required according to national regulations. Informed consent was not applicable.

Consent for publication

Not applicable.

\section{Competing interests}

The authors declare that they have no competing interests.

Received: 25 June 2019 Accepted: 18 February 2020

Published online: 22 February 2020

\section{References}

1. Lee BE, Davies HD. Aseptic meningitis. Curr Opin Infect Dis. 2007;20(3):272-7.

2. Costerus JM, et al. Community-acquired bacterial meningitis. Curr Opin Infect Dis. 2017;30(1):135-41.

3. McGill F, Griffiths MJ, Solomon T. Viral meningitis: current issues in diagnosis and treatment. Curr Opin Infect Dis. 2017:30(2):248-56.

4. Almuhayawi $M$, et al. Identification of microorganisms by FilmArray and matrix-assisted laser desorption ionization-time of flight mass spectrometry prior to positivity in the blood culture system. J Clin Microbiol. 2014;52(9): 3230-6.

5. Fitch MT, van de Beek D. Emergency diagnosis and treatment of adult meningitis. Lancet Infect Dis. 2007;7(3):191-200.

6. Leber $\mathrm{AL}$, et al. Multicenter evaluation of BioFire FilmArray meningitis/ encephalitis panel for detection of Bacteria, viruses, and yeast in cerebrospinal fluid specimens. J Clin Microbiol. 2016;54(9):2251-61.

7. Tarai B, Das P. FilmArray $(R)$ meningitis/encephalitis (ME) panel, a rapid molecular platform for diagnosis of CNS infections in a tertiary care hospital in North India: one-and-half-year review. Neurol Sci. 2019;40(1):81-8.

8. Nestor D, et al. Evaluation of the FilmArray meningitis/encephalitis panel with focus on bacteria and Cryptococcus spp. J Microbiol Methods. 2019; 157:113-6.

9. Launes $C$, et al. Utility of FilmArray meningitis/encephalitis panel during outbreak of brainstem encephalitis caused by Enterovirus in Catalonia in 2016. J Clin Microbiol. 2017;55(1):336-8.

10. Graf EH, Farquharson MV, Cardenas AM. Comparative evaluation of the FilmArray meningitis/encephalitis molecular panel in a pediatric population. Diagn Microbiol Infect Dis. 2017:87(1):92-4.

11. Arora HS, et al. Enhanced identification of group B streptococcus and Escherichia Coli in young infants with meningitis using the Biofire Filmarray meningitis/encephalitis panel. Pediatr Infect Dis J. 2017;36(7):685-7.

12. Wootton $\mathrm{SH}$, et al. Enhancing pathogen identification in patients with meningitis and a negative gram stain using the BioFire FilmArray((R)) meningitis/encephalitis panel. Ann Clin Microbiol Antimicrob. 2016;15:26.

13. Blaschke AJ, et al. Retrospective Evaluation of Infants Aged 1 to 60 Days with Residual Cerebrospinal Fluid (CSF) Tested Using the FilmArray Meningitis/Encephalitis (ME) Panel. J Clin Microbiol. 2018;56(7):e00277-18.

14. Gomez CA, et al. Delayed Diagnosis of Tuberculous Meningitis Misdiagnosed as Herpes Simplex Virus-1 Encephalitis With the FilmArray Syndromic Polymerase Chain Reaction Panel. Open Forum Infect Dis. 2017; 4(1):ofw245

15. Dien Bard J, Alby K. Point-Counterpoint: Meningitis/Encephalitis Syndromic Testing in the Clinical Laboratory. J Clin Microbiol. 2018;56(4):e00018.

16. Kniehl E, Dörries H, Geiss RK, Matz B, Neumann-Häfelin D, Pfister HW, Prange H, Schlüter B, Spellerberg FB. Spencker MIQ 17: Qualitätsstandards in der 
mikrobiologisch-infektiologischen Diagnostik: Infektionen des Zentralnervensystems; 2001.

17. Abdeldaim G, et al. Usefulness of real-time PCR for lytA, ply, and Spn9802 on plasma samples for the diagnosis of pneumococcal pneumonia. Clin Microbiol Infect. 2010;16(8):1135-41.

18. Abdeldaim GM, et al. Multiplex quantitative PCR for detection of lower respiratory tract infection and meningitis caused by streptococcus pneumoniae, Haemophilus influenzae and Neisseria meningitidis. BMC Microbiol. 2010;10:310

19. Benschop K, et al. Rapid detection of human parechoviruses in clinical samples by real-time PCR. J Clin Virol. 2008;41(2):69-74.

20. Cassina $G$, et al. Calibrated real-time polymerase chain reaction for specific quantitation of HHV-6A and HHV-6B in clinical samples. J Virol Methods. 2013;189(1):172-9.

21. Diaz MH, et al. Optimization of multiple pathogen detection using the TaqMan Array card: application for a population-based study of neonatal infection. PLoS One. 2013;8(6):e66183.

22. Dierssen $U$, et al. Rapid routine detection of enterovirus RNA in cerebrospinal fluid by a one-step real-time RT-PCR assay. J Clin Virol. 2008; 42(1):58-64

23. Gatto F, et al. A multiplex calibrated real-time PCR assay for quantitation of DNA of EBV-1 and 2. J Virol Methods. 2011;178(1-2):98-105.

24. Khansarinejad B, et al. Quantitation of human cytomegalovirus DNA in plasma using an affordable in-house qPCR assay. J Virol Methods. 2012; 183(2):170-5.

25. Le Monnier A, et al. Diagnosis of listeria monocytogenes meningoencephalitis by real-time PCR for the hly gene. J Clin Microbiol. 2011;49(11):3917-23

26. Meylan $\mathrm{S}$, et al. Real-time PCR for type-specific identification of herpes simplex in clinical samples: evaluation of type-specific results in the context of CNS diseases. J Clin Virol. 2008:41(2):87-91.

27. Pollak $\mathrm{L}$, et al. Varicella zoster vs. herpes simplex meningoencephalitis in the PCR era. A single center study. J Neurol Sci. 2012;314(1-2):29-36.

28. Ryschkewitsch CF, Jensen PN, Major EO. Multiplex qPCR assay for ultra sensitive detection of JCV DNA with simultaneous identification of genotypes that discriminates non-virulent from virulent variants. J Clin Virol. 2013;57(3):243-8.

29. Si-Mohamed A, et al. Detection and quantitation of BK virus DNA by realtime polymerase chain reaction in the LT-ag gene in adult renal transplant recipients. J Virol Methods. 2006;131(1):21-7.

30. Stralin K, et al. Comparison of sputum and nasopharyngeal aspirate samples and of the PCR gene targets lytA and Spn9802 for quantitative PCR for rapid detection of pneumococcal pneumonia. J Clin Microbiol. 2014;52(1): 83-9.

31. Veron $\mathrm{V}$, et al. Real-time polymerase chain reaction detection of Cryptococcus neoformans and Cryptococcus gattii in human samples. Diagn Microbiol Infect Dis. 2009;65(1):69-72.

32. Radmard $\mathrm{S}$, et al. Clinical utilization of the FilmArray meningitis/encephalitis (ME) multiplex polymerase chain reaction (PCR) assay. Front Neurol. 2019;10:281.

33. Naccache SN, et al. One Year in the Life of a Rapid Syndromic Panel for Meningitis/Encephalitis: a Pediatric Tertiary Care Facility's Experience. J Clin Microbiol. 2018;56(5):e01940-17.

34. Hanson KE, et al. Preclinical assessment of a fully automated multiplex PCR panel for detection of central nervous system pathogens. J Clin Microbiol. 2016;54(3):785-7.

35. Messacar $\mathrm{K}$, et al. Potential clinical impact of the film array meningitis encephalitis panel in children with suspected central nervous system infections. Diagn Microbiol Infect Dis. 2016;86(1):118-20.

36. Anand $\mathrm{V}$, et al. Closing the brief case: neonatal meningitis caused by listeria monocytogenes diagnosed by multiplex molecular panel. J Clin Microbiol. 2016;54(12):3075.

37. Anand $V$, et al. The brief case: neonatal meningitis caused by listeria monocytogenes diagnosed by multiplex molecular panel. J Clin Microbiol. 2016;54(12):2846-9.

38. Lumley SF, et al. Multiplex PCR reveals high prevalence of enterovirus and HHV6 in acellular paediatric cerebrospinal fluid samples. J Inf Secur. 2018; 77(3):249-57.

39. Brouwer MC, Tunkel AR, van de Beek D. Epidemiology, diagnosis, and antimicrobial treatment of acute bacterial meningitis. Clin Microbiol Rev. 2010;23(3):467-92.
40. Negre VL, et al. Culture-negative neonatal meningitis and endocarditis caused by Streptococcus agalactiae. J Clin Microbiol. 2004:42(10):4889-90.

41. Lu JJ, et al. Use of PCR with universal primers and restriction endonuclease digestions for detection and identification of common bacterial pathogens in cerebrospinal fluid. J Clin Microbiol. 2000;38(6):2076-80.

42. Kristiansen $\mathrm{BE}$, et al. Rapid diagnosis of meningococcal meningitis by polymerase chain reaction. Lancet. 1991;337(8757):1568-9.

43. Ericsdotter AC, et al. Reactivation of herpes simplex type 1 in pneumococcal meningitis. J Clin Virol. 2015;66:100-2.

44. Labska $\mathrm{K}$, et al. Presence of herpesvirus DNA in cerebrospinal fluid of patients with tick-borne encephalitis and enteroviral meningoencephalitis. J Med Virol. 2015;87(7):1235-40.

45. Green DA, et al. Clinical significance of human Herpesvirus 6 positivity on the FilmArray meningitis/encephalitis panel. Clin Infect Dis. 2018;67(7):1125-8.

46. Slenker AK, Royer TL, Villalobos T. Human herpes virus 6 positivity on the FilmArray meningitis/encephalitis needs clinical interpretation. Clin Infect Dis. 2019;69:192-4.

47. Soucek DK, et al. Cost Justification of the BioFire FilmArray Meningitis/ Encephalitis Panel Versus Standard of Care for Diagnosing Meningitis in a Community Hospital. J Pharm Pract. 2017;32:36-40. https://doi.org/10.1177/ 0897190017737697.

48. Duff S, et al. Economic analysis of rapid multiplex polymerase chain reaction testing for meningitis/encephalitis in pediatric patients. Future Microbiol. 2018;13:617-29.

49. Patel R, Fang FC. Diagnostic stewardship: opportunity for a laboratoryinfectious diseases partnership. Clin Infect Dis. 2018;67(5):799-801.

50. Tan NW, et al. Cerebrospinal fluid white cell count: discriminatory or otherwise for enteroviral meningitis in infants and young children? J NeuroOncol. 2016;22(2):213-7.

51. Eichinger $A$, et al. Clinical benefits of introducing real-time multiplex PCR for cerebrospinal fluid as routine diagnostic at a tertiary care pediatric center. Infection. 2019;47(1):51-8.

\section{Publisher's Note}

Springer Nature remains neutral with regard to jurisdictional claims in published maps and institutional affiliations.

Ready to submit your research? Choose BMC and benefit from:

- fast, convenient online submission

- thorough peer review by experienced researchers in your field

- rapid publication on acceptance

- support for research data, including large and complex data types

- gold Open Access which fosters wider collaboration and increased citations

- maximum visibility for your research: over $100 \mathrm{M}$ website views per year

At $\mathrm{BMC}$, research is always in progress.

Learn more biomedcentral.com/submissions 